\title{
Management of Germ Cell Tumours of the Testes in Adult Patients: German Clinical Practice Guideline, PART II - Recommendations for the Treatment of Advanced, Recurrent, and Refractory Disease and Extragonadal and Sex Cord/Stromal Tumours and for the Management of Follow-Up, Toxicity, Quality of Life, Palliative Care, and Supportive Therapy
}

\author{
Sabine Kliesch ${ }^{\mathrm{a}}$ Stefanie Schmidt ${ }^{\mathrm{b}}$ Doris Wilborn ${ }^{\mathrm{b}}$ Clemens Aigner ${ }^{\mathrm{c}}$ Walter Albrecht $^{\mathrm{d}}$ \\ Jens Bedke ${ }^{\mathrm{e}}$ Matthias Beintker ${ }^{\mathrm{f}}$ Dirk Beyersdorffg ${ }^{\mathrm{C}}$ Carsten Bokemeyer ${ }^{\mathrm{h}}$ Jonas Busch ${ }^{\mathrm{i}}$ \\ Johannes Classen ${ }^{j}$ Maike de Wit ${ }^{k}$ Klaus-Peter Dieckmann' ${ }^{\prime}$ Thorsten Diemer ${ }^{m}$ Anette Dieing ${ }^{n}$ \\ Matthias Gockel $^{\circ}$ Bernt Göckel-Beining ${ }^{p}$ Oliver W. Hakenberg ${ }^{q} \quad$ Axel Heidenreich $^{r}$ \\ Julia Heinzelbecker ${ }^{\mathrm{S}}$ Kathleen Herkommer ${ }^{\mathrm{t}}$ Thomas Hermanns $^{\mathrm{u}}$ Sascha Kaufmann ${ }^{v}$ \\ Marko Kornmann ${ }^{\text {w Jörg Kotzerke }}{ }^{\mathrm{x}}$ Susanne Krege ${ }^{\mathrm{y}}$ Glen Kristiansen ${ }^{z}$ Anja Lorch ${ }^{\mathrm{A}}$ \\ Arndt-Christian Müller $^{v}$ Karin Oechsle ${ }^{h}$ Timur Ohloff $^{B}$ Christoph Oing $^{\text {h }}$ Ulrich Otto $^{C}$ \\ David Pfister $^{r}$ Renate Pichler ${ }^{D}$ Heinrich Recken ${ }^{E}$ Oliver Rick ${ }^{F}$ Yvonne Rudolph ${ }^{G}$ \\ Christian Ruf $^{\mathrm{H}}$ Joachim Schirren' Hans Schmelz' Heinz Schmidberger ${ }^{\mathrm{K}}$ Mark Schrader $^{\mathrm{L}}$ \\ Stefan Schweyer $^{\mathrm{M}}$ Stefanie Seeling ${ }^{\mathrm{N}}$ Rainer Souchon ${ }^{\mathrm{V}}$ Christian Winter $^{\mathrm{O}}$ Christian Wittekind $^{\mathrm{P}}$ \\ Friedemann Zengerling ${ }^{Q} \quad$ D.H. Zermann ${ }^{R}$ Roger Zillmann ${ }^{S}$ Peter Albers ${ }^{A}$ \\ ${ }^{a}$ Centre of Reproductive Medicine and Andrology, Department of Clinical and Surgical Andrology, University Hospital \\ Münster, Münster, Germany; ${ }^{b}$ UroEvidence, Deutsche Gesellschaft für Urologie, Berlin, Germany; ${ }^{c}$ Ruhrlandklinik, \\ University Hospital Essen, Essen, Germany; ${ }^{\mathrm{d}}$ Department of Urology, Landesklinikum Mistelbach-Gänserndorf, \\ Mistelbach, Austria; ${ }^{e}$ Department of Urology, University Hospital Tübingen, Tübingen, Germany; ${ }^{f}$ Südharz Hospital \\ Nordhausen gGmbH, Nordhausen, Germany; ${ }^{9}$ Clinic and Polyclinic for Diagnostic and Interventional Radiology and

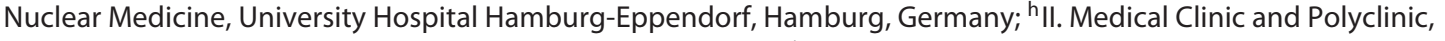 \\ University Hospital Hamburg-Eppendorf, Hamburg, Germany; 'Department of Urology, Charité University Hospital, \\ Berlin, Germany; 'Department of Radiotherapy, Radiological Oncology, and Palliative Medicine, St. Vincentius-Kliniken, \\ Karlsruhe, Germany; ${ }^{k}$ Clinic for Internal Medicine - Hematology, Oncology, and Palliative Medicine, Vivantes Clinic \\ Neukölln, Berlin, Germany; 'Department of Urology, Asklepios Klinik Altona, Hamburg, Germany; ${ }^{m}$ Clinic and Polyclinic \\ for Urology, Pediatric Urology, and Andrology, University Hospital Gießen, Gießen, Germany; ${ }^{\mathrm{n} C l i n i c}$ for Internal \\ Medicine - Hematology and Oncology, Vivantes Clinics Am Urban, Berlin, Germany; ${ }^{\circ}$ HELIOS Clinics Berlin-Buch,



 \\ Urology, University Hospital Saarland, Saarbrücken, Germany;
}

S. Kliesch and S. Schmidt contributed equally to this study and should be considered co-first authors.

karger@karger.com www.karger.com/uin

Karger $\stackrel{\text { ' }}{5}$

BOPEN ACCESS
C) 2021 The Author(s)

Published by S. Karger AG, Basel

This is an Open Access article licensed under the Creative Commons Attribution-NonCommercial-4.0 International License (CC BY-NC) (http://www.karger.com/Services/OpenAccessLicense), applicable to the online version of the article only. Usage and distribution for commercial purposes requires written permission.
Peter Albers

Universitätsklinikum Düsseldorf

Gebäude 13.71/72, Moorenstraße 5

DE-40225 Düsseldorf (Germany)

p.albers@dkfz-heidelberg.de 
tUrological Clinic and Polyclinic of the Technical University of Munich, Munich, Germany; u'Department of Urology, University Hospital Zurich, University of Zurich, Zurich, Switzerland; 'Department for Radiooncology, University

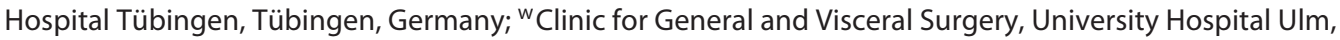
Ulm, Germany; ${ }^{x}$ University Hospital Dresden, Dresden, Germany; ${ }^{y} \mathrm{KEM}$, Protestant Hospital Essen-Mitte, Clinic for Urology, Pediatric Urology and Urological Oncology, Essen, Germany; ${ }^{2}$ Institute of Pathology, University Hospital Bonn, Bonn, Germany; ${ }^{A}$ Department of Urology, Universitätsklinikum Düsseldorf, Düsseldorf, Germany; ${ }^{B}$ German Foundation for Young Adults with Cancer, Berlin, Germany; ${ }^{C}$ Urological Competence Centre for Rehabilitation, Bad Wildungen, Germany; ${ }^{D}$ Department of Urology, Medical University Innsbruck, Innsbruck, Austria; ${ }^{E}$ Hamburger Fern-Hochschule Studienzentrum Essen, Essen, Germany; ${ }^{F}$ Klinik Reinhardshöhe GmbH, Bad Wildungen, Germany; ${ }^{G}$ German Cancer Society, Berlin, Germany; ${ }^{H}$ Department of Urology, Bundeswehrkrankenhaus, Ulm, Germany; 'Helios Clinics, Wiesbaden, Germany; ${ }^{\mathrm{J}}$ Department of Urology, Bundeswehrkrankenhaus, Koblenz, Germany; ${ }^{\mathrm{K}} \mathrm{Clinic}$ and Polyclinic for Radiooncology and Radiotherapy, University Hospital Mainz, Mainz, Germany; ${ }^{L}$ Helios Clinics,

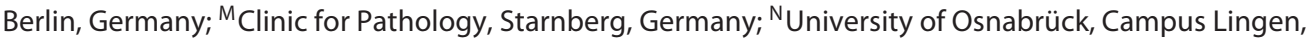
Osnabrück, Germany; ${ }^{O}$ Urologie Neandertal, Erkrath, Germany; ${ }^{P}$ Institute of Pathology, University Hospital Leipzig,

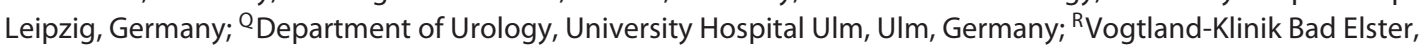
Bad Elster, Germany; ${ }^{S}$ Urology Pankow, Berlin, Germany

\section{Keywords}

Germ cell tumour of the testes - Seminoma .

Non-seminoma - Metastasis - Extragonadal tumours .

Stromal tumours - Therapy · Follow-up - Toxicity ·

Quality of life · Palliative care · Supportive therapy ·

Systematic review - Guideline

\begin{abstract}
Objectives: We developed the first German evidence- and consensus-based clinical guideline on diagnosis, treatment, and follow-up of germ cell tumours (GCT) of the testes in adult patients. We present the guideline content in 2 separate publications. The present second part summarizes the recommendations for the treatment of advanced disease stages and for the management of follow-up and late effects. Materials and Methods: An interdisciplinary panel of $42 \mathrm{ex}-$ perts including 1 patient representative developed the guideline content. Clinical recommendations and statements were based on scientific evidence and expert consensus. For this purpose, evidence tables for several review questions, which were based on systematic literature searches (last search in March 2018), were provided. Thirty-one experts, who were entitled to vote, rated the final clinical recommendations and statements. Results: Here we present the treatment recommendations separately for patients with metastatic seminoma and non-seminomatous GCT (stages IIA/B and IIC/III), for restaging and treatment of residual masses, and for relapsed and refractory disease stages. The recommendations also cover extragonadal and sex
\end{abstract}

cord/stromal tumours, the management of follow-up and toxicity, quality-of-life aspects, palliative care, and supportive therapy. Conclusion: Physicians and other medical service providers who are involved in the diagnostics, treatment, and follow-up of GCT (all stages, outpatient and inpatient care as well as rehabilitation) are the users of the present guideline. The guideline also comprises quality indicators for measuring the implementation of the guideline recommendations in routine clinical care; these data will be presented in a future publication.

(c) 2021 The Author(s)

Published by S. Karger AG, Basel

\section{Background}

We are presenting the main content of the new German clinical practice guideline in a publication series; part I covers the following chapters: epidemiology, classification systems, diagnostics, prognosis, and treatment recommendations for the localized stages [1]. Here, part II covers the recommendations for the metastatic stages, for recurrent and refractory disease, and for follow-up, toxicity management, quality of life, palliative care, and supportive therapy.

The online supplementary German S3 guideline (see www.karger.com/doi/10.1159/000511245 for all online suppl. material) is freely available for download in the German language in both a short and a long version (http://www.awmf.org/leitlinien/detail/1l/043-044. html). 


\section{Objective}

The objectives of this guideline have been described previously in part I [1].

\section{Methods}

The methods of this guideline have been described previously in part I [1].

\section{Results}

Chapter 8: Primary and First-Line Therapy

(Continued)

See part I for the previous recommendations [1]. See also Tables 1-3 for the dosages and applications.

\section{Metastatic Germ Cell Tumours of the Testes} Stages IIA/B

19 In patients with cSIIA seminoma either radiotherapy or chemotherapy with 3 cycles of BEP (bleomycin, etoposide, and cisplatin; alternatively, 4 cycles of EP [etoposide and cisplatin] if bleomycin is contraindicated) is recommended. The lack of prospective randomized controlled trials for the direct comparison of radiotherapy versus chemotherapy for seminoma cSIIA/B does not allow a valid statement regarding the superiority or equivalence in oncological efficacy of either therapy. We recommend informing the patient about the advantages and disadvantages of both treatment options (grade A: very low quality of the evidence) [e2-e6].

20 It is recommended that patients with cSIIB seminoma receive chemotherapy with 3 cycles of $\mathrm{BEP}$ or, in case of contraindications to bleomycin, 4 cycles of EP. Alternatively, radiotherapy can be performed. The lack of prospective randomized controlled trials for the direct comparison of radiotherapy versus chemotherapy for seminoma cSIIB does not allow a valid statement regarding the superiority or equivalence in oncological efficacy of either therapy. We recommend informing the patient about the advantages and disadvantages of both treatment options (grade A: very low quality of the evidence) [e2, e4, e6].

21 Radiotherapy with $30 \mathrm{~Gy}$ (total dose) is recommended for patients with cSIIA seminoma and radiotherapy with $36 \mathrm{~Gy}$ (total dose) is recommended in stage cSIIB seminoma $(A, 2 b)$.
Table 1. BEP regimen

\begin{tabular}{lll}
\hline Substance & Dosage & Application \\
\hline Cisplatin & $20 \mathrm{mg} / \mathrm{m}^{2}(1$ h i.v. $)$ & Days $1-5$ \\
Etoposide & $75-100 \mathrm{mg} / \mathrm{m}^{2}(1$ h i.v. $)$ & Days $1-5$ \\
Bleomycin & $30 \mathrm{mg}$ (bolus i.v.) & Days 1,8 , and \\
& & 15
\end{tabular}

Repeat on day 22.

Table 2. EP regimen

\begin{tabular}{lll}
\hline Substance & Dosage & Application \\
\hline Etoposide & $100 \mathrm{mg} / \mathrm{m}^{2}(1$ h i.v. $)$ & Days $1-5$ \\
Cisplatin & $20 \mathrm{mg} / \mathrm{m}^{2}(1$ h i.v. $)$ & Days $1-5$ \\
\hline
\end{tabular}

Repeat on day 22.

Table 3. VIP regimen

\begin{tabular}{lrl}
\hline Substance & \multicolumn{1}{l}{ Dosage } & Application \\
\hline Cisplatin & $20 \mathrm{mg} / \mathrm{m}^{2}$ (1 h i.v. $)$ & Days 1-5 \\
Etoposide & $100 \mathrm{mg} / \mathrm{m}^{2}$ (1 h i.v.) & Days 1-5 \\
Ifosfamide & $1,200 \mathrm{mg} / \mathrm{m}^{2}$ (2 h i.v.) & Days 1-5 \\
\hline
\end{tabular}

Repeat on day 22.

22 We recommend performing a control imaging with an abdominal/pelvic CT scan 2-3 months after radiotherapy. It is recommended to proceed equally after chemotherapy. The result of this examination is also the starting point for follow-up care (expert consensus).

23 In non-seminomatous cSIIA/B GCT we recommend treatment according to the International Germ Cell Cancer Collaborative Group (IGCCCG) with chemotherapy (3-4 cycles of BEP), and in case of a residual tumour we recommend treatment with residual tumour resection (RTR). Exception: in GCT initially classified as $100 \%$ teratoma in the orchiectomy specimen without a marker increase, retroperitoneal lymph node dissection (RPLND) is recommended $(A, 2 b)[\mathrm{e}$, e8].

24 Patients with serum tumour marker-negative nonseminomatous cSIIA GCT should be monitored first (serum tumour marker monitoring, repeat imaging af- 
ter 6-8 weeks) or, alternatively, a diagnostic RPLND should be performed $(B, 2 b)$ [e7, e8].

25 In patients with serum tumour marker-negative cSIIA non-seminomatous GCT and after primary RPLND with $\mathrm{pN} 1$ findings (PS IIA) in the R0 status, active surveillance is recommended $(A, 1 b)$. Alternatively, $2 \mathrm{cy}$ cles of EP can be administered if there is no pure teratoma (0) [e9-e12, e7].

26 Patients after primary RPLND in serum tumour marker-negative cSIIA non-seminomatous GCT with pN2 findings (PS IIB) should be monitored in the R0 status $(B, 1 b)$. Alternatively, if there is no pure teratoma, 2 cycles of BEP or EP can be administered (0) [e13, e9, e10, e12, e7].

\section{Stages IIC/III}

27 The standard regimen of polychemotherapy for metastatic disease is BEP (statement, 1b) [e9].

28 Growth factors should be used in BEP as a secondary prophylaxis in case of neutropenic fever in the previous cycle $(B, 1 b)$ [e9, e14, e15].

29 When applying the VIP regimen (etoposide, ifosfamide, cisplatin), we recommend using a myeloid growth factor (G-CSF) as a primary prophylaxis $(A$, 1b) $[\mathrm{e} 14, \mathrm{e} 16]$.

30 In patients with metastatic IIC/III stage GCT of the good prognosis group according to the IGCCCG, we recommend polychemotherapy with 3 cycles of BEP with application of cisplatin and etoposide for 5 days $(A, 1 b)$ [e17-e19, e15].

31 In patients with acute life-threatening metastatic gonadal GCT, we recommend primarily administering chemotherapy without a prior orchiectomy (expert consensus).

32 After completion of chemotherapy in patients with gonadal GCT, we recommend performing an orchiectomy of the initially affected testis if this has been postponed due to acute life-threatening advanced metastatic disease at diagnosis $(A, 4)$ [e20-e23].

33 In patients with stage IIC/III metastatic seminoma and a good prognosis we recommend 3 cycles of BEP chemotherapy $(A, 1 a)$ [e24].

34 In patients with stage IIC/III metastatic seminoma and a good prognosis we recommend 4 cycles of EP if bleomycin is contraindicated $(A, 4)$ [e25, e26].

35 In patients with metastatic seminoma and an intermediate prognosis, we recommend 4 cycles of $\operatorname{BEP}(A, 1 b)$.

36 In patients with metastatic seminoma and an intermediate prognosis, we recommend 4 cycles of VIP if bleomycin is contraindicated $(A, 1 b)$ [e27].
37 In patients with metastatic non-seminomatous GCT stage IIC/III and a good prognosis, we recommend 3 cycles of $\operatorname{BEP}(A, 1 b)$ [e18].

38 Patients with metastatic non-seminomatous GCT stage IIC/III and a good prognosis should receive 4 cycles of EP if contraindications to bleomycin exist $(B$, 1b) [e18, e28].

39 In patients with metastatic non-seminomatous GCT and an intermediate prognosis we recommend 4 cycles of $\operatorname{BEP}(A, 1 b)$ [e27].

40 In patients with metastatic non-seminomatous GCT and an intermediate prognosis, we recommend $4 \mathrm{cy}$ cles of VIP if contraindications to bleomycin exist $(A$, 1b) [e27].

41 In patients with metastatic non-seminomatous GCT and a poor prognosis, we recommend 4 cycles of BEP $(A, 1 b)$ [e9].

42 In patients with metastatic non-seminomatous GCT and a poor prognosis, we recommend 4 cycles of VIP if contraindications to bleomycin exist $(A, 1 b)$ [e29].

43 In patients with primary mediastinal disease, bone and/or brain metastases, and/or an inadequate serum tumour marker decline, we recommend considering treatment intensification $(A, 1 b-4)$ [e30-e34].

$44 \mathrm{It}$ is recommended that the evaluation of treatment intensification be performed at a centre with proven experience (expert consensus).

45 We do not recommend general intensification of firstline therapy in favour of a high-dose chemotherapy with autologous stem cell transplantation for the unselected group of all GCT patients with a poor prognosis $(A, 1 b)$ [e35, e36].

46 To assess the response to therapy, we recommend analysis of the serum tumour marker decline after 1 , or at the latest after 2, cycles of standard-dose chemotherapy $(A, 1 b)$ [e35].

47 In patients with an inadequate serum tumour marker decline after 1 or 2 cycles of cisplatin-based first-line therapy, intensification of chemotherapy should be considered on an individual basis (grade B: moderate quality of the evidence) [e37].

48 When chemotherapy is intensified by means of primary high-dose therapy, sequential therapy should be performed with 3 cycles of high-dose VIP, each followed by autologous stem cell transplantation $(B, 1 b)$ [e38, e36].

See also Table 4 [e36]. 
Table 4. High-dose VIP regimen (after e36)

\begin{tabular}{lrl}
\hline PEB & & \\
$\begin{array}{l}\text { Cisplatin } \\
\text { Etoposide }\end{array}$ & $20 \mathrm{mg} / \mathrm{m}^{2}(1$ h i.v. $)$ & Days $1-5$ \\
Ifosfamide & $75 \mathrm{mg} / \mathrm{m}^{2}(30 \mathrm{~min}$ to 1 h i.v. $)$ & $\begin{array}{l}\text { Days } 1-5 \\
\text { Days } 1-5\end{array}$ \\
\hline $\begin{array}{l}\text { High-dose } P E B \\
\text { Cisplatin }\end{array}$ & $200 \mathrm{mg} / \mathrm{m}^{2}(2$ h i.v. $)$ & \\
$\begin{array}{l}\text { Etoposide } \\
\text { Ifosfamide }\end{array}$ & $300 \mathrm{mg} / \mathrm{m}^{2}(1$ h i.v. $)$ & Days $1-5$ \\
& $2,400 \mathrm{mg} / \mathrm{m}^{2}(2$ h i.v. $)$ & Days $1-5$ \\
\hline
\end{tabular}

High-dose VIP: stem cell re-infusion on day 7.

Special Considerations

49 In patients with brain metastases at the initial diagnosis, we recommend either referral of patients to a GCT centre with proven experience or consultation at a centre with proven expertise prior to therapy (expert consensus).

50 In patients with brain metastases at the initial diagnosis, we recommend 4 cycles of chemotherapy (BEP, VIP) according to the IGCCCG poor prognosis group $(A, 2 b)$. Alternatively, primary therapy intensification can be performed (0) [e30, e39, e40, e33].

51 Due to insufficient data, no reliable statement regarding the benefit of post-chemotherapeutic radiotherapy and/or resection (in case of residual brain metastasis*). Any additional treatment should be decided upon based on interdisciplinary discussion on an individual basis (statement, 2b) [e30, e39, e40, e15, e33].

52 Due to documented long-term toxicity of whole brain radiotherapy of the neurocranium, stereotactic radiotherapy should be sought if possible $(B, 2 b)[\mathrm{e} 39, \mathrm{e} 40$, e33].

53 In patients with bone metastases at the initial diagnosis, we recommend 4 cycles of chemotherapy (BEP, VIP) according to the IGCCCG intermediate prognosis (seminoma) and poor prognosis (non-seminomatous GCT) group $(A, 4)[\mathrm{e} 34]$.

$54 \mathrm{We}$ recommend that patients with bone metastases at the initial diagnosis be referred to a centre with proven experience, or that such a centre be consulted prior to therapy (expert consensus).

55 Primary high-dose chemotherapy is no standard approach (in patients with bone metastases*), but it should be evaluated on an individual basis and considered where appropriate $(B, 2 b)$ [e32].

$56 \mathrm{We}$ recommend considering subsequent local therapy of bone metastases following chemotherapy on an in-

German Clinical Practice Guideline on

GCT of the Testes dividual basis (resection or radiation if technically possible). Due to insufficient data, a reliable statement regarding the benefit of post-chemotherapeutic radiotherapy and/or resection of residual bone lesions cannot be provided $(A, 4)$ [e34, e41].

$57 \mathrm{We}$ recommend performing decompression of tumour-induced hydronephrosis before initiating chemotherapy (expert consensus).

58 In patients with renal impairment, we do not recommend general replacement of cisplatin by carboplatin. The individual treatment decision should be made after consulting a centre with proven experience or a second-opinion online portal such as eKonsil Urologie (Hodentumor) in Germany (https://hodentumor. zweitmeinung-online.de/) (expert consensus).

59 Carboplatin-based chemotherapy is associated with increased recurrence rates and increased tumour-related mortality and it should therefore be restricted to justifiable individual cases (statement, 1b) [e42-e44].

60 We recommend that patients with active HIV disease receive sufficient antiretroviral therapy before initiating chemotherapy. It is recommended that the choice of antiretroviral therapy consider possible interactions with the cytostatic agents applied (expert consensus).

61 Active HIV disease is not a contraindication to stagematched chemotherapy. Primary prophylactic administration of G-CSF (unless it is with VIP therapy) and antibiotic prophylaxis are not required (expert consensus).

62 In patients with active HIV disease*, we recommend determining the CD4 cell count before each chemotherapy cycle. With CD 4 counts $<200 / \mu \mathrm{L}$, antibiotic prophylaxis with cotrimoxazole should be administered (expert consensus).

63 Patients with advanced/metastatic GCT disease should preferably be treated by multidisciplinary teams at centres with proven experience in order to maximize the chance of cure and prevent treatment-associated adverse events by avoiding overtreatment (expert consensus).

* Additional information due to the abbreviated presentation of the text.

Restaging and Treatment of Residual Tumour Disease 64 Immediate chemotherapy without a prior orchiectomy should only be initiated in patients with life-threatening symptoms and high serum tumour markers for AFP and $\beta$-hCG $(B, 4)$ [e45].

65 FDG-PET/CT can be applied in seminoma patients with normal or normalized serum tumour markers 
who have residual disease larger than $3 \mathrm{~cm}$ in diameter after completion of therapy $(0,1 a)$. The $\mathrm{CT}$ as part of the FDG-PET/CT scan should preferably be performed as a contrast-enhanced CT (expert consensus) [e46, e47].

$66 \mathrm{We}$ recommend performing FDG-PET/CT for the evaluation of serum tumour marker-negative residual tumours in seminoma patients not earlier than 6 weeks after completion of chemotherapy (expert consensus).

67 In case of positive metabolic activity in FDG-PET/CT, intensified follow-up (CT and serum tumour marker control) should be performed (expert consensus).

68 After completion of first-line therapy of a metastatic seminoma, RTR should not be performed $(B, 1 b)$ [e48].

69 Restaging after first-line chemotherapy should not include an FDG-PET/CT scan in patients with non-seminomatous $\operatorname{GCT}(B, 2 b)$ [e49, e50].

70 In patients with non-seminomatous GCT, we recommend resection of residual retroperitoneal and pulmonary tumours $>1 \mathrm{~cm}$ that persist after completion of first-line chemotherapy and achievement of serum tumour marker normalization. It is recommended that the management of residual tumours at other sites be decided upon individually $(A, 2 b)$ [e51].

71 Surgical removal of residual pulmonary metastases $>1$ $\mathrm{cm}$ is recommended. It is recommended that bilateral thoracic RTR be decided on interdisciplinarily when the histology of the resection of the first side is available. It is recommended that the resection be carried out using a parenchyma-saving approach with the opportunity for digital palpation $(A, 4)$ [e52, e53].

72 It is recommended that RTR be performed in centres with proven experience with the opportunity for interdisciplinary surgical care (e.g., liver resections, vascular grafting, spinal neurosurgery) $(A, 2 b)$ [e54-e56].

73 Retroperitoneal RTR should preferably be performed using a nerve-sparing technique $(B, 4)$. It is recommended that the template of RTR be defined depending on the initial tumour site $(A)$ [e57-e59].

74 Tumour resection only (so-called lumpectomy) should not be performed $(B, 2 b)$ [e57, e60-e63].

75 In patients with R0 resection upon RTR, follow-up is recommended. In case of vital tumour cells, an individual approach is recommended, depending on the histology, the postoperative serum tumour markers, and morbidity, since a benefit for survival after adjuvant chemotherapy is currently unclear (expert consensus).

76 In patients after salvage chemotherapy of a retroperitoneal non-seminomatous GCT relapse, retroperito- neal RTR should be performed even in tumours $<1 \mathrm{~cm}$ if tumour markers have normalized $(B, 4)$ [e64].

\section{Chapter 9: Recurrent and Refractory Tumours}

Recurrences after the Initial Stage I

1 In patients with recurrent seminoma after initial cSI, we recommend classification and treatment according to the extent of disease based on IGCCCG criteria (expert consensus).

2 In patients with a recurrent non-seminomatous GCT after the initial stage I, we recommend classification and treatment based on IGCCCG criteria. Only in patients with recurrence earlier than 6 months after adjuvant therapy with 2 cycles of BEP should alternative chemotherapy regimens be considered (expert consensus).

Recurrence after Chemotherapy for Metastatic Disease

3 We recommend classification of patients with recurrence after first-line chemotherapy for metastatic disease according to the risk classification of the International Prognostic Factors Study Group (IPFSG) (expert consensus).

4 Patients with recurrence after cisplatin-based combination chemotherapy due to primary metastatic disease can receive conventional-dose chemotherapy according to the TIP (paclitaxel, ifosfamide, cisplatin) or VIP regimen $(0,2 b)$ [e65].

5 Patients after cisplatin-based combination chemotherapy for primary metastatic disease should receive primary salvage chemotherapy using sequential highdose chemotherapy with 3 cycles of HD-CE (carboplatin + etoposide) and autologous stem cell support ( $B$, 1b). Alternatively, patients with relapse following cisplatin-based combination chemotherapy for primary metastatic disease can receive conventional-dose chemotherapy according to the TIP or VIP regimen (0) [e66-e68].

6 Patients (with recurrence*) after cisplatin-based combination chemotherapy for primary metastatic disease should be included in clinical trials if possible (expert consensus).

7 In non-seminomatous GCT patients undergoing salvage chemotherapy for a retroperitoneal relapse, RTR should be performed even in residual tumours $<1 \mathrm{~cm}$ after normalization of tumour markers $(B, 4)$ [e64].

* Additional information due to the abbreviated presentation of the text.
Kliesch et al. 
Patients with Bone Metastases at Recurrence

8 In patients with recurrent bone metastases, we recommend performing a systemic combination chemotherapy (expert consensus).

9 Since bone metastases at recurrence represent a highrisk situation, the patient should preferentially be treated with high-dose chemotherapy (expert consensus).

10 After systemic therapy of recurrent bone metastases, additional local therapy (resection or radiotherapy) should always be evaluated. Comparative prospective data are not available for these two therapy options (expert consensus).

11 For vitality assessment after systemic chemotherapy, a biopsy can be considered prior to local therapy in patients with extensive bone metastases (expert consensus).

Cerebral Metastases in Recurrence

12 In patients with cerebral metastases at relapse, highdose chemotherapy should be administered $(B, 2 b)$. Retrospective studies indicate a survival benefit from consolidation radiotherapy. In case of incomplete remission, it is recommended that radiotherapy be performed additionally $(A, 2 b)$. In case of complete remission of cerebral metastases in imaging after salvage chemotherapy, the situation is unclear and additive radiotherapy should be considered individually $(B, 2 b)$ [e33].

13 In patients with solitary residuals after salvage chemotherapy, we recommend performing stereotactic radiation and/or neurosurgical resection of brain metastases (expert consensus).

14 In patients with multiple residuals after salvage chemotherapy, it is recommended that radiotherapy of the neurocranium be performed (expert consensus).

Late Relapses

15 In patients with marker-negative late recurrence and in whom complete resectability seems possible, we recommend performing a primary surgical resection $(A$, 2b) [e69, e70].

16 Patients with a non-resectable late recurrence have a poor prognosis. These patients should primarily receive chemotherapy (either conventional or high-dose chemotherapy) and secondary resection should be considered $(B, 4)[\mathrm{e} 71]$.
Refractory and Multiply Recurrent GCT

17 In patients with recurrence or progressive relapse after multiple chemotherapy regimens, we recommend offering a combination chemotherapy with gemcitabine and oxaliplatin $+/$ - paclitaxel (GOP). Following a response to therapy with GOP, the resection of all residual tumours is recommended $(A, 4)$ [e72-e77].

\section{Special Cases}

18 In patients with growing teratoma syndrome, we recommend complete surgical resection $(A, 4)$ [e78-e80].

19 In cSI patients with malignant somatic transformation, we recommend $\operatorname{RPLND}(A, 4)[\mathrm{e} 81, \mathrm{e} 82]$.

20 In patients with metastatic disease and components of malignant somatic transformation, we recommend performing a GCT-based chemotherapy first, followed by RPLND (expert consensus).

21 In patients with malignant somatic transformation relapsing after GCT therapy, we recommend choosing a chemotherapy regimen based on the histology of the somatic malignancy $(A, 4)[\mathrm{e} 83, \mathrm{e} 81, \mathrm{e} 82]$.

\section{Chapter 10: GCT Not Related to GCNIS and Sex Cord/Stromal Tumours Spermatocytic Tumour}

1 The spermatocytic tumour - formerly spermatocytic seminoma - is a very rare tumour that requires differentiation from classical seminoma. The diagnostic measures do not differ from those applied in gonadal GCT (expert consensus).

2 The pure spermatocytic tumour limited to the testes is a tumour with a good prognosis, which is sufficiently treated by orchiectomy and surveillance (expert consensus).

3 The presence of sarcomatous components in a spermatocytic tumour indicates a highly malignant transformation of the tumour with a poor prognosis and requires a multimodal therapy concept with chemotherapy, RTR, and possibly radiation therapy. The sequence of the individual steps needs to be determined individually for each patient (expert consensus).

4 It is recommended that sarcomatous components of the spermatocytic tumour be ruled out upon histological evaluation (expert consensus).

\section{Extragonadal GCT}

5 Ninety-five percent of all male adult GCT originate in the testicles; $5 \%$ are of primarily extragonadal origin (expert consensus). 
6 We recommend confirming the diagnosis of extragonadal GCT histologically only in case of an ambiguous constellation of serum tumour markers (expert consensus).

7 The determination of the serum tumour markers (AFP, $\beta$-hCG, LDH) in extragonadal GCT is recommended for confirmation of the diagnosis, for correct classification according to the IGCCCG, and for monitoring of the treatment responses (expert consensus).

8 All patients with mediastinal non-seminomatous extragonadal GCT are classified as belonging to the poor prognosis group (stage III) according to the IGCCCG. Due to their very poor prognosis, high-dose chemotherapy should be chosen primarily. Performance of a radical RTR should yield a good response $(B, 2 b)$. We recommend the involvement of centres with proven experience in the therapy $(A, 2 b)$ [e84].

9 We recommend to classify patients with retroperitoneal non-seminomatous extragonadal GCT depending on their serum tumour marker levels according to the IGCCCG classification $(A, 2 b)$ [e84].

$10 \mathrm{We}$ recommend performing therapy of extragonadal GCT in analogy to metastatic gonadal GCT according to the histology and the corresponding IGCCCG classification. Centres with proven experience should be involved in the therapy (expert consensus).

\section{Sex Cord/Stromal Tumours}

11 In patients suspected of having a Leydig cell tumour or a Sertoli cell tumour, we recommend determining the serum tumour markers (AFP, $\beta$-hCG, LDH) as well as testosterone, $\mathrm{LH}$, and FSH for endocrinological evaluation (expert consensus).

12 We recommend establishing intraoperatively the histological diagnosis by frozen section. We recommend performing organ-preserving surgery in small tumours $(<2 \mathrm{~cm})$ if no malignancy criteria are present $(A, 3)[\mathrm{e} 85]$.

13 Malignant gonadal stroma tumours of Leydig cell or Sertoli cell origin should be treated with radical orchiectomy (expert consensus).

14 If histological examination of Leydig cell or Sertoli cell tumours reveals criteria for the malignancy, RPLND should be performed in stage I patients $(B, 2 b)$ [e54, e86, e87].

15 In malignant Leydig cell or Sertoli cell tumours we recommend performing an RPLND in stage II patients (expert consensus).

\section{Chapter 11: Follow-Up after Curative Therapy}

There is no international consensus on follow-up recommendations for patients with GCT in full remission after curative therapy. A number of publications address follow-up schedules.

1 MRI of the abdomen/pelvis should replace CT of the abdomen/pelvis in the follow-up care of patients with GCT if performed at centres with proven experience to reduce radiation exposure. Experienced radiologists are required for the interpretation of results (expert consensus).

The recommendations given in Tables 5-11 are based on the following publications and are separately illustrated for the different patient groups [e88-e90].

\section{Chapter 12: Toxicity, Management of Toxicity, and Supportive Measures}

1 Acute toxicity occurring during therapy should be recorded systematically and appropriate therapeutic measures should be taken (expert consensus).

2 In the follow-up care, the examination and, if necessary, the treatment of therapy-related late sequelae and long-term toxicity play a central role in addition to the search for disease recurrence (expert consensus).

3 After radiation or chemotherapy, fertility impairment is common in GCT patients compared to the normal male population and can persist as a long-term sequela after completion of treatment (expert consensus).

4 GCT patients are at risk of clinically relevant hypogonadism. Therefore, we recommend asking the patient about related symptoms and determining serum levels of testosterone and $\mathrm{LH}$ as part of the diagnostic measures during therapy and follow-up (expert consensus).

5 In patients with hypogonadism, therapy follows the recommendations for testosterone replacement therapy (expert consensus).

6 During cisplatin-containing chemotherapy for metastatic GCT, thromboembolism prophylaxis with lowmolecular-weight heparin should be used to reduce the risk of thromboembolic events in patients $(B, 2 b)$ [e91].

\section{Chapter 13: Quality of Life and Rehabilitation}

1 In patients with GCT, quality-of-life data should be collected before and during treatment (expert consensus).

2 The EORTC QLQ-TC 26 questionnaire for quality-oflife assessment, which was specifically developed for patients with GCT, is available in the German language (statement, $3 b$ ) [e92]. 
Table 5. Group 1: patients with local therapy in the retroperitoneum

\begin{tabular}{llccc}
\hline Histology & Stage & Therapy & $\begin{array}{c}\text { Recurrence } \\
\text { rate }(\%)\end{array}$ & $\begin{array}{l}\text { Occurrence of } \\
\text { recurrence }\end{array}$ \\
\hline Seminoma & I & $20 \mathrm{~Gy}$ & $3-5$ & Years 1-3 \\
Seminoma & IIA & $30 \mathrm{~Gy}$ & $5-10$ & Years 1-4 \\
Seminoma & IIB & $36 \mathrm{~Gy}$ & $10-15$ & Years 1-4 \\
Non-seminomatous GCT & IIA/B & $3 \times \mathrm{PEB}+\mathrm{RTR}$ & $2-5$ & Years 1-3 \\
Non-seminomatous GCT & IIC/III (good prognosis) & $3 \times \mathrm{PEB}+\mathrm{RTR}$ & $11-18$ & Years 1-4 \\
\hline
\end{tabular}

Table 6. Follow-up schedule for group 1: patients with local therapy in the retroperitoneum

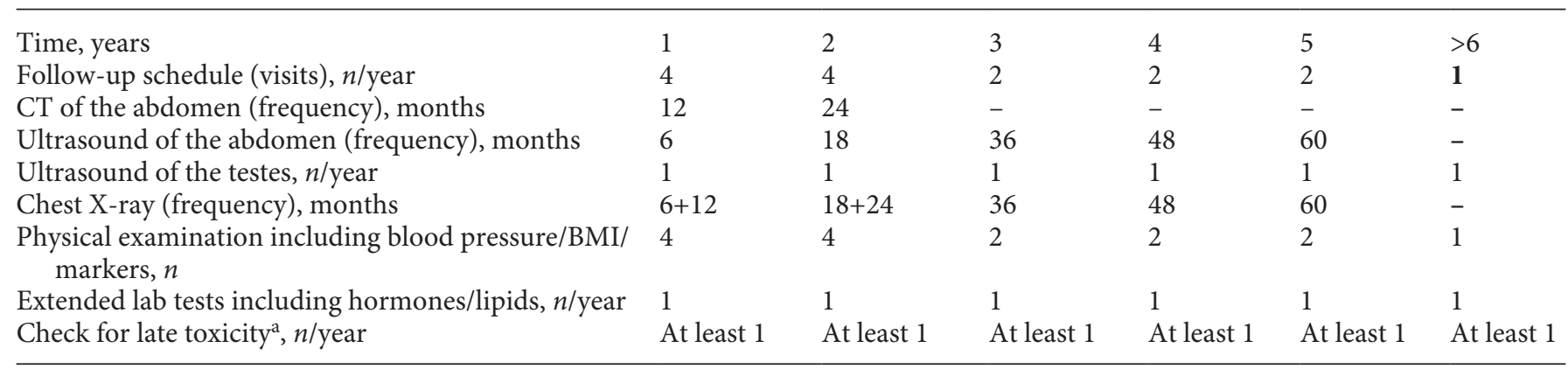

Seminoma CS I, IIa/b + radiotherapy, non-seminomatous GCT IIa/b + 3x PEB + RTR, non-seminomatous GCT IIc/III good + 3x $\mathrm{PEB}+\mathrm{RTR}$, including CT of the pelvis in seminoma stage I after radiotherapy. In non-seminomatous GCT with initial supradiaphragmal involvement (stage III) instead of chest X-ray: chest CT at 6, 12, and 24 months. ${ }^{a}$ Intervals need to be adapted to the respective therapy.

Table 7. Group 2: Patients without local therapy in the retroperitoneum

\begin{tabular}{|c|c|c|c|c|}
\hline Seminoma & I & Carbo monotherapy & $3-5$ & Years $1-3^{b}$ \\
\hline Seminoma $^{a}$ & IIB/IIC/III (good prognosis) & $3 \times \mathrm{PEB} / 4 \mathrm{EP}$ & $11-18$ & Years 1-4 \\
\hline Non-seminomatous GCT & I high risk & $\begin{array}{l}1 \times \mathrm{PEB} \\
\text { (or } 2 \times \mathrm{PEB} \text { according to the } \\
\text { former recommendation) }\end{array}$ & $3-5$ & Years 1-5 \\
\hline Non-seminomatous GCT & IIA/B/IIC/III (good prognosis) & $3 \times \mathrm{PEB}$ & $8-18$ & Years 1-3 \\
\hline
\end{tabular}

${ }^{\text {a }}$ Complete remission or residual tumour $<3$ or $>3 \mathrm{~cm}$, PET negative. ${ }^{\mathrm{b}} 15 \%$ of seminoma recurrences still occur after the $3 \mathrm{rd}$ followup year.

3 Depending on individual needs, it is recommended that all patients eligible for rehabilitation be offered post-treatment rehabilitation (expert consensus).
4 It is recommended that the rehabilitation of patients with GCT be carried out in rehabilitation clinics offering age-specific services with oncological competence and by using multimodal therapy concepts (expert consensus). 
Table 8. Follow-up schedule for group 2: patients without local therapy in the retroperitoneum

Time, years

Follow-up schedule (visits), $n$ /year

CT of the abdomen (frequency) ${ }^{\mathrm{a}}$, months

Ultrasound of the abdomen (frequency), months

Ultrasound of the testes, $n /$ year

Chest X-ray (frequency), months

Physical examination of blood pressure/BMI/markers, $n$

Extended lab tests including hormones/lipids, $n /$ year

Check for late toxicity ${ }^{\mathrm{b}}, n /$ year

$\begin{array}{llllll}1 & 2 & 3 & 4 & 5 & >6 \\ 4 & 4 & 2 & 2 & 2 & 1 \\ 6+12 & 24 & - & - & - & - \\ (6 \mathrm{~b}) & 18 & 36 & 48 & 60 & - \\ 1 & 1 & 1 & 1 & 1 & 1 \\ 6+12 & 18+24 & 36 & 48 & 60 & - \\ 4 & 4 & 2 & 2 & 2 & 1 \\ 1 & 1 & 1 & 1 & 1 & 1 \\ \text { At least } 1 & \text { At least } 1 & \text { At least } 1 & \text { At least 1 } & \text { At least 1 } & \text { At least 1 }\end{array}$

Seminoma I + 1× carbo, seminoma IIA/IIB/C, III good + 3× PEB/4 EP, non-seminomatous GCT I high $+2 \times$ PEB, non-seminomatous GCT IIA-C, III good $+3 \times$ PEB. No CT in month 6 for seminoma and non-seminomatous GCT stage I after chemotherapy, but ultrasound of the abdomen. In non-seminomatous GCT with initial supradiaphragmal involvement (stage III) instead of a chest X-ray: chest CT at 6,12 , and 24 months. ${ }^{a} 15 \%$ of recurrences in stage I seminoma still occur after 3 years, so that follow-up with CT of the abdomen may have to be adjusted. ${ }^{\mathrm{b}}$ Intervals must be adapted to the respective therapy.

Table 9. Follow-up schedule for group 3: patients under active surveillance

\begin{tabular}{lllll}
\hline Group & Histology/stage & Therapy & Recurrence rate, \% & Period of time \\
\hline $3 \mathrm{~A}^{\mathrm{a}}$ & Seminoma I & None & $12-31$ & Years 1 and 2 \\
$3 \mathrm{~B}^{\mathrm{b}}$ & Non-seminomatous GCT (low risk) & None & $14-22$ & Years 1 and 2 \\
\hline
\end{tabular}

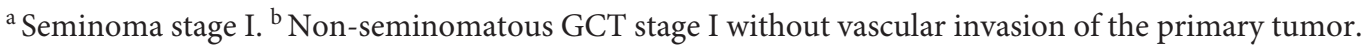

Table 10. Follow-up schedule for group 3A: seminoma stage I under active surveillance

\begin{tabular}{|c|c|c|c|c|c|c|}
\hline Time, years & 1 & 2 & 3 & 4 & 5 & $6-10$ \\
\hline Follow-up schedule (visits), $n /$ year & 4 & 4 & 2 & 2 & 2 & 1 \\
\hline CT of the abdomen (frequency), months & $6+12$ & $18+24$ & - & - & - & - \\
\hline Ultrasound of the abdomen (frequency), months & $3+9$ & $15+21$ & $30+36$ & 48 & 60 & - \\
\hline Ultrasound of the testes, $n /$ year & 1 & 1 & 1 & 1 & 1 & 1 \\
\hline Chest X-ray (frequency), months & $6+12$ & $18+24$ & 36 & 48 & 60 & - \\
\hline Clinical examination of blood pressure/BMI/markers, $n$ & 4 & 4 & 2 & 2 & 2 & 1 \\
\hline Extended lab tests including hormones/lipids, $n /$ year & 1 & 1 & 1 & 1 & & 1 \\
\hline
\end{tabular}

Table 11. Follow-up schedule for group 3B: nonseminomatous GCT stage I low-risk tumours under active surveillance

\begin{tabular}{|c|c|c|c|c|c|c|}
\hline Time, years & 1 & 2 & 3 & 4 & 5 & $6-10$ \\
\hline Follow-up schedule (visits), $n /$ year & 6 & 6 & 4 & 2 & 2 & 1 \\
\hline CT of the abdomen (frequency), months & $4+12$ & - & - & - & - & - \\
\hline Ultrasound of the abdomen (frequency), months & - & 24 & 36 & 48 & 60 & - \\
\hline Chest X-ray (frequency), months & $6+12$ & $18+24$ & $30+36$ & 48 & 60 & - \\
\hline Clinical examination including blood pressure/BMI/markers, $n$ & 6 & 6 & 4 & 2 & 2 & 1 \\
\hline Extended lab tests including hormones/lipids, $n /$ year & 1 & 1 & 1 & 1 & 1 & 1 \\
\hline
\end{tabular}


Chapter 14: Palliative Care, Psycho-Oncology, and Supportive Therapy

1 If incurable disease becomes obvious, we recommend informing the patient and, when required, his or her relatives, at an early stage about palliative care options. Access to these should be facilitated (expert consensus).

2 Low-threshold psychosocial counselling, support, and treatment should be available to all affected persons and their relatives in every phase of the disease, as well as in the long term (expert consensus).

\section{Key Messages of Part II}

- The lack of prospective randomized controlled trials for the direct comparison of radiotherapy versus chemotherapy for seminoma cSIIA/B does not allow a valid statement regarding the superiority or equivalence of either therapy with respect to oncological efficacy.

- In patients with acute life-threatening disease with metastatic gonadal GCT in stages IIC/III, we recommended administerimng chemotherapy upfront and postponing orchiectomy of the initially affected testis until completion of the chemotherapy.

- We recommend analysis of the serum tumour markers after 1 , or at the latest after 2, cycles of standard-dose chemotherapy.

- Particular attention and careful consideration are required in patients with contraindications to bleomycin, with brain and bone metastases, with renal impairment, or with active HIV infection.

- In patients with primary mediastinal disease, bone and/or brain metastases, and/or an inadequate serum tumour marker decline, we recommend evaluating treatment intensification.

- We recommend classifying and treating patients with relapsing seminoma or non-seminomatous GCT after the initial stage I based on their recurrent stage by analogy to primary metastatic seminoma/non-seminomatous GCT according to IGCCCG criteria.

- There is no international consensus on follow-up recommendations for patients with GCT in full remission after curative therapy.

- In patients with a serum tumour marker-negative late recurrence, we recommend performing a primary surgical resection in cases where complete resectability is technically feasible.

- In patients with relapse or progression after multiple chemotherapy, we recommend offering a combination chemotherapy with gemcitabine and oxaliplatin $+/-$ pa- clitaxel (GOP). Following a response to therapy with GOP, resection of all residual tumours is recommended.

- Acute toxicity occurring during therapy should be recorded systematically and appropriate therapeutic measures should be taken.

- In the follow-up care, the examination, and, if necessary, the treatment of therapy-related late sequelae and long-term toxicity play a central role in addition to the search for disease recurrence.

- In patients with GCT, quality-of-life data should be collected before and during treatment.

- Research gaps in the context of this guideline were particularly evident for healthcare research and with respect to risk factors for disease incidence. In addition, the statements related to the poor prognosis group are based on limited data. The evaluation of follow-up schedules by long-term studies is also still pending.

\section{Acknowledgement}

The German Guideline Program in Oncology of the Association of the Medical Scientific Societies (AWMF), the German Cancer Society (DKG), and the German Cancer Aid Foundation $(\mathrm{DKH})$ supervised this project and gave methodological support. Administrative support by Ingrid Rambow (Münster, Germany) was greatly appreciated. Uwe Hölzel (Dresden, Germany) also participated in this project.

\section{Conflict of Interest Statement}

The evaluation of conflicts of interests showed only minor relevance on the topic and can be obtained via request.

\section{Funding Sources}

Support was received from the DKH (reference No. 70112789).

\section{Author Contributions}

All of the authors have made substantial contributions to the conception or design of this work and the acquisition, analysis, and interpretation of data for this work. They drafted this work or revised it critically for important intellectual content and approved the final version to be published. They have agreed to be accountable for all aspects of this work in ensuring that questions related to the accuracy or integrity of any part of this work are appropriately investigated and resolved.

\section{References}

1 Kliesch S, et al. Management of germ cell tumours of the testis in adult patients. German clinical practice guideline PART I: Epidemiology, classification, diagnosis, prognosis, fertility preservation and treatment recommendations for localized stages. Urol Int. Forthcoming 2020. 